\title{
INVESTIGATION OF PRODUCTION OUTPUT FOR IMPROVEMENT
}

\section{SALAWU ENESI YEKINI, OKOKPUJIE IMHADE P., S. A AFOLALU, AJAYI O. O \& AZETA JOSEPH}

Department of Mechanical Engineering, Covenant University, Ota, Ogun State, Nigeria

\begin{abstract}
Experimental determination and evaluation of ten (10) different production lines producing different brands and sizes of alcohol beverages was carried out on each of the lines according to the standard operating procedures (SOP) of the machine operation, processes and quality inspection standard. Operation was carried out for 2 shifts of 12 hours each for 26 days in the month of September, 27 days in October, and 25 days each for the month of November and December. The set target for each of the lines were used as critical control limit for each line, monthly operation and the monthly output for each line was recorded by converging the result of the daily production for each month. The result from the plot revealed that line 10 performed better than every other line in September and October with average output per hour of 126.6 cases and 106.9 cases respectively, while line 9 performed better in the month of November with an average output per hour of 1113 cases followed by line 8 with an average output per hour of 108.8 cases in December. The experimental analysis had really revealed the reasons behind the poor performances of each line for each month hence there is need for performance improvement for increased productivity.
\end{abstract}

KEYWORDS: Experimental Evaluation, Operation Management, Machine Operation, S O P \& Target

Received: Dec 04, 2017; Accepted: Dec 23, 2017; Published: Jan 31, 2018; Paper Id.: IJMPERDFEB2018111

\section{INTRODUCTION}

Production management is the process of transforming various resources in the operations subsystem of the organization into value added product in a controlled manner as per the policies of the organization. Therefore, it is part of an organization, which is concerned with the transformation of a range of inputs into the required product having the requisite quality level to assess and improve on the performance of the production plant. Daan Van Leer [1] said that Heineken needs to stay ahead on the competitive market and needs to constantly improve the product performance. The research work focused on a new production line which produces star bottles that differs from other production line because of its multiple speeds on the filling machine. This line is a self regulated production line where speed levels of the machines are regulated by sensors on the production line. The overall performance of this line was found to be below the target, hence the need for improvement was necessary to achieved this, is by making a study of the process analysis in order to consider every part of the production line as well as analyzing the data to detect the possible failures of the production line. Rui et al [2] applied lean manufacturing techniques in a food and beverage industries to improve their productivity by sensitizing the employees on the importance of lean concepts and the implementation. The employees were informed and trained on the $5 \mathrm{~S}$ methodology on several work stations. In this work, a detailed analysis was made on each of the work stations, evaluating the organization of equipment and which tools and documents were needed to perform most common operations. Finally gave corrective measures which consist of tidying up, organizing, cleaning, normalizing and establishing control measures in work stations. An evaluation checklist was performed again and was set as a routine task to instill a continuous improvement in their production activities.

Fawaz et al [3] analyzed the benefits of lean manufacturing and value stream mapping via simulation in a 
process sector by applying lean techniques in a large integrated steel mill. Value stream mapping and simulation model were used to make comparison between the "before" and "after" scenarios in detail so that the managers can actually see the benefits such as reduced production lead-time and lower work-in-process. Detailed simulation model was developed for the current system and later modified to model the proposed future state and validated to ensure that the simulation model mimic the real system. Jafri et al [4] also applied lean fundamental principles to construct a VSM for identification and elimination of wastes by team formation, product selection, conceptual design, and time-frame formulation through take time calculation. Based on the future VSM, the final results showed that by implementing some lean thinking techniques, Production Lead-time (PLT) decreased from 8.5 days to 6 days, and the value added time decreased from 68 minutes to 37 minutes. Sibson [5] used a novel methodology to investigate the performance of production line systems using an integrated product service system approach. The approach involves modeling and simulation techniques based on reliability engineering principles and systems thinking. Systems thinking approach was employed to create the static simulation model of the integrated production line system by means of schematic representations. Key improvement areas, identified from the static simulation model were modeled dynamically to incorporate the stochastic behaviour of the system. Dynamic models were developed using a Discrete Event Simulation platform. These models were supported by Monte Carlo Simulation, queuing principles, probabilistic and statistical methods pertinent to reliability engineering.

Malgorzota et al [6] did a work on improving the performance of a bottling plant based on simulation by building a discrete event simulation using data provided by maintenance department, which identified two major failures in the system and approximated those data using continuous statistical tools. The model was also validated by taking measures of line performance and evaluations. Further failure analysis was done using pareto charts to check for areas which need improvement and concluded that the simulated model that useful decisions can be made during maintenance decision making. According to Mike Poland [7], line performance optimization is a problem-solving technique in pharmaceutical industry which is usually done by creating a value stream mapping methodology for the manufacturing process. Analysis of the current-state value stream map and ways to improve material flow, eliminate non-value-added activities and as well optimize the manual and automated processes. It is also important to evaluate the mean time between failures and the mean time to repair for the sensitive machines to determine the need to develop failure mode and effect analysis. This will enable us to develop a future-state map that reflects the improvement we have identified.

Colledani et al [8] did a Performance evaluation of linear and non-linear multi-product, multi-stage lines with unreliable machines and finite homogeneous buffers, by assuming that machines flexibilities can be improved to produce different products with the same method by having fast and accurate method to evaluate the system performance. Analytical method was proposed for the performance evaluation of production systems in which $\mathrm{Z}$ different types of product were produced. The proposed method was based on decomposition of complex systems into a set of building blocks in which each one was formed by two -machines and $\mathrm{Z}$ homogenous buffers. Further more analyzed the set of building blocks model as the behavior of the original system. The performance of each building block was evaluated using the aggregate technique applied to two-machine lines in which the behavior of several products is modeled by an aggregate technique. This further illustrates the complexity of the analysis and the numerical result proved that his method provides accurate results.

Tomasz et al [9] used a discrete event simulation tool to reduce the negative influence of plant and machinery failures on the performance of a filling line. The packaging line consisted of seven machines which were connected by 
conveyors. Utilized the collected data from machine failures to model and simulate using FlexiSim software. Then introduced buffers in the production system which resulted in increased in output by $15 \%$. According to Riddhish et al [10], Six sigma is a quality initiative which reduces variations in a process and helps to lower the cost of product as well as process and examined the advancement encounters of six sigma practices in Global manufacturing Industries, and identified the key tools for each step in successful Six Sigma project execution. The work also applied the lessons learned from successful Six Sigma projects and their applications in manufacturing industries.

\section{Brief Description of the Bottling Processes}

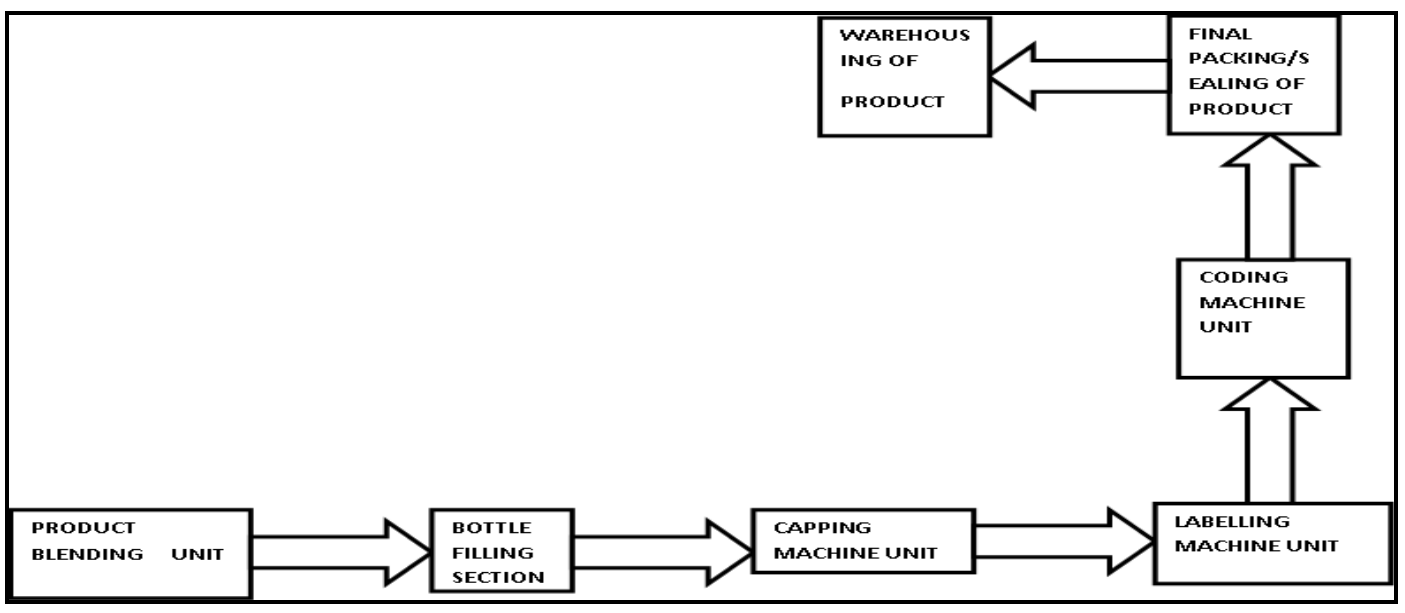

Figure 1: Layout of Alcohol Beverage Packaging Line

A production line can be defined as a system in which product visit a number of workstations in a stable sequential manner. The workstation is also a subsystem in production which consists of one or more machines where product processing takes place Figure 1 show the layout of alcohol beverage packaging line.

\section{PROBLEM DEFINITION}

Productivity is not just about total output achieved during operation or production, but also a function of how effective and efficient are the materials utilized in achieving the output. Production performance optimization is therefore important in operation management. However, line performance cannot improve when only the losses are known, there must be a practical measure to put in place to avert or reduce the losses to a minimal level in order to increase productivity. Once losses can be determined, machine line efficiency and relationship can then be determined and improved on. Over time the company in study has been experiencing low output and great losses as well. Situations arises daily on the machines resulting to high losses of the material during operation, therefore there is need to assess and know the performances of each of the lines and also compare the output with the expected target and identify the major problems responsible for low output. The aim of the paper is to study the performances of each of the production line, to identify and address the root causes of poor output of the machines that arise during the bottling process. Also suggest possible measures to the identified problems.

\section{MATERIALS AND METHODS}

The production process was carried out on each of the lines according to the standard operating procedures of line operation and quality standard management. Ten (10) lines production activities were experimented from September to 
December and each of the line activities was monitored for 12 hours for each working day, the output for each month was then collated by converging the daily production output for that month. The percentage output delivered for each of the month was also calculated and documented. The production line in study is an alcohol processing and packaging line. The machine arrangements followed a linear pattern and are linked together by means of a stainless steel chain conveyor according to the process flow. When the blending process of the alcohol beverage was completed, the required product to be bottled was supplied through stainless pipe connections to the filler machine. The filler machine operator took some sample of the supplied product to the laboratory for product parameter analysis before full bottling commenced. The product was certified okay by the quality control department, full bottling was then carried out.

The bottles were moved to the next work station which is a capper which performed the functions of sealing and threading of the cap head on the bottle to ensure that it was airtight. The products were transported to the next work station where it was coded with the batch number and production date for record tracking purposes. The next work station was the labeler which also did the labeling of the product and passed to the final packaging unit where the products were finally packaged into their respective cartons and sealed manually, for the case of pet bottles, sealing machines were used. The monthly target for each of the lines were noted and recorded, the number of days worked were also recorded.

\section{RESULTS AND DISCUSSIONS}

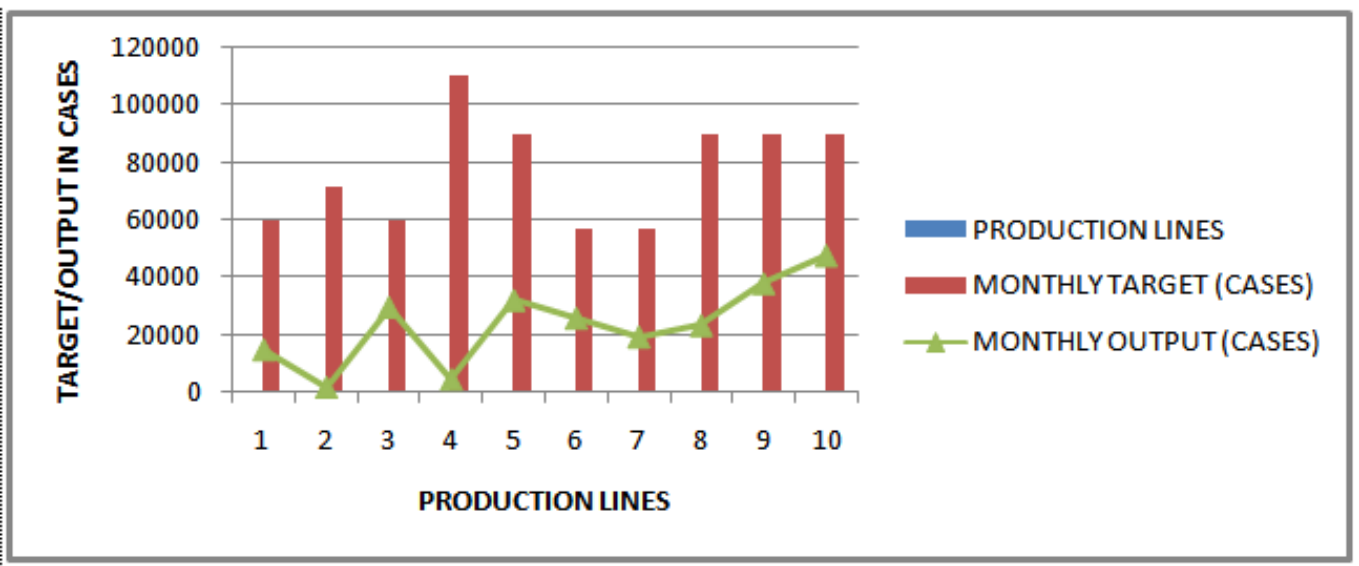

Figure 2: Expected Target/Output for Each Line for the Month of September 2016

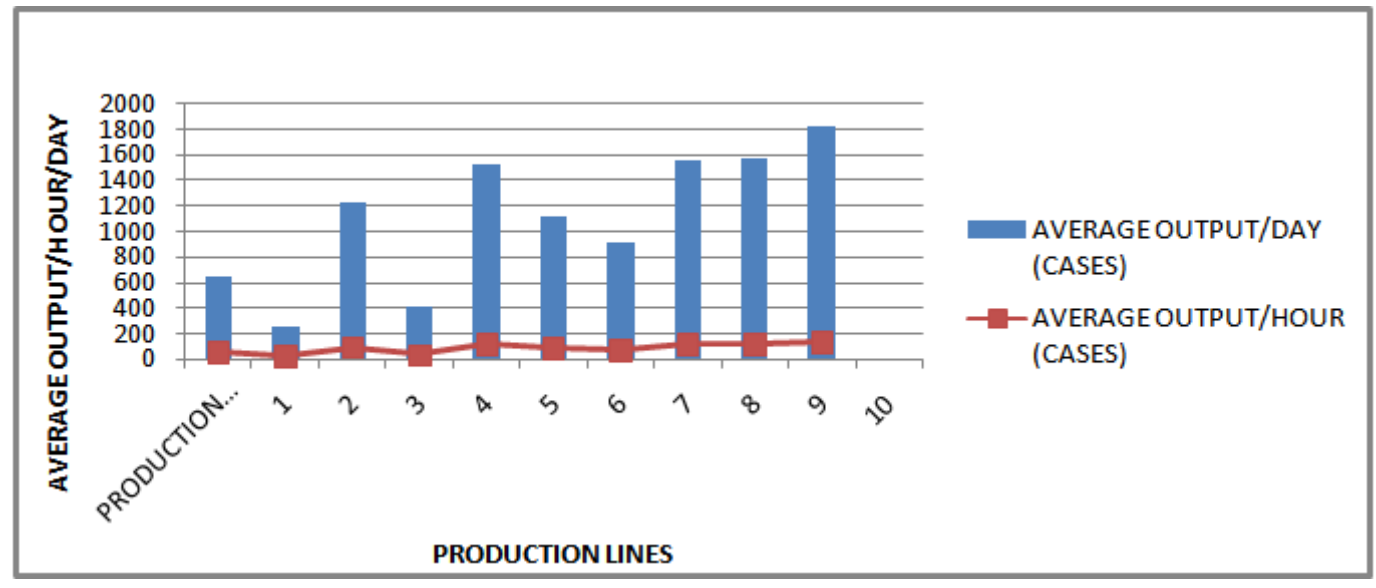

Figure 3: Average Production for September 2016

Figure 2 and 3 presents the plots of set target/expected output and average production/hour/day respectively for 
the same period. Production was carried out on each line for a period of 26 days the result from the two plots shows that line L10 performed better than every other line with an average production output of 126.6 cases per hour, though far below target. Line 2, 4 and 1 had a very low output of 17.4, 28.4 and 44.5cases per hour. This was as a result of frequent failures from the filler machine star wheels, worm drive and labeler off centering problem recorded in the loss time report.

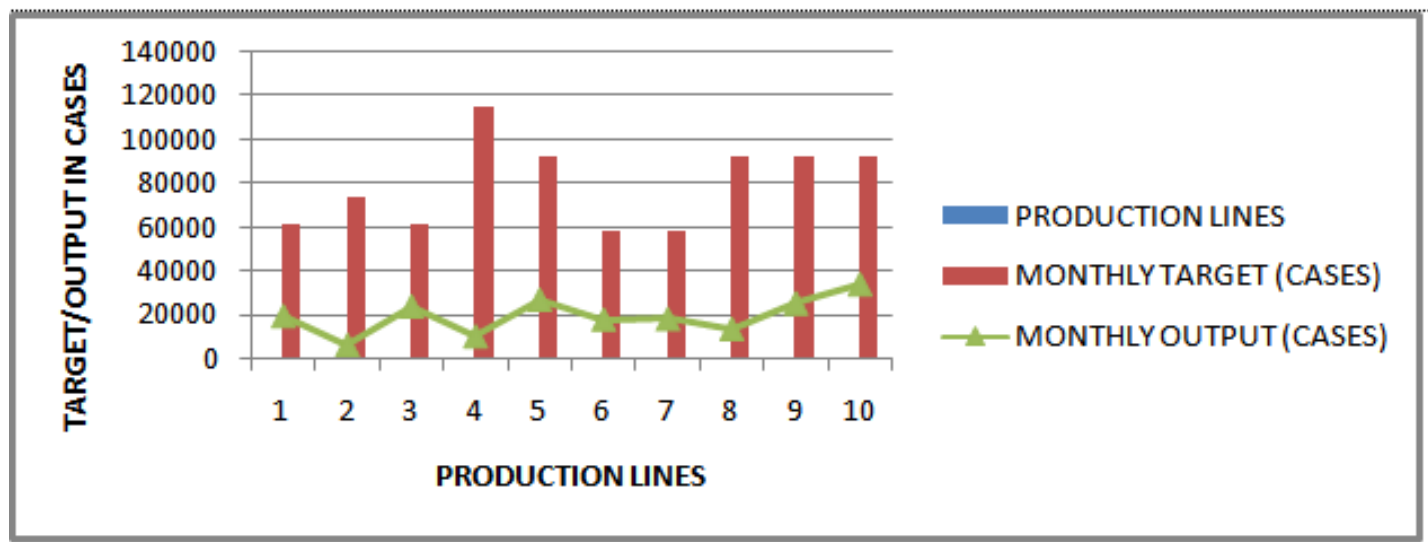

Figure 4: Expected Target/Output for Each Line for the Month of October 2016

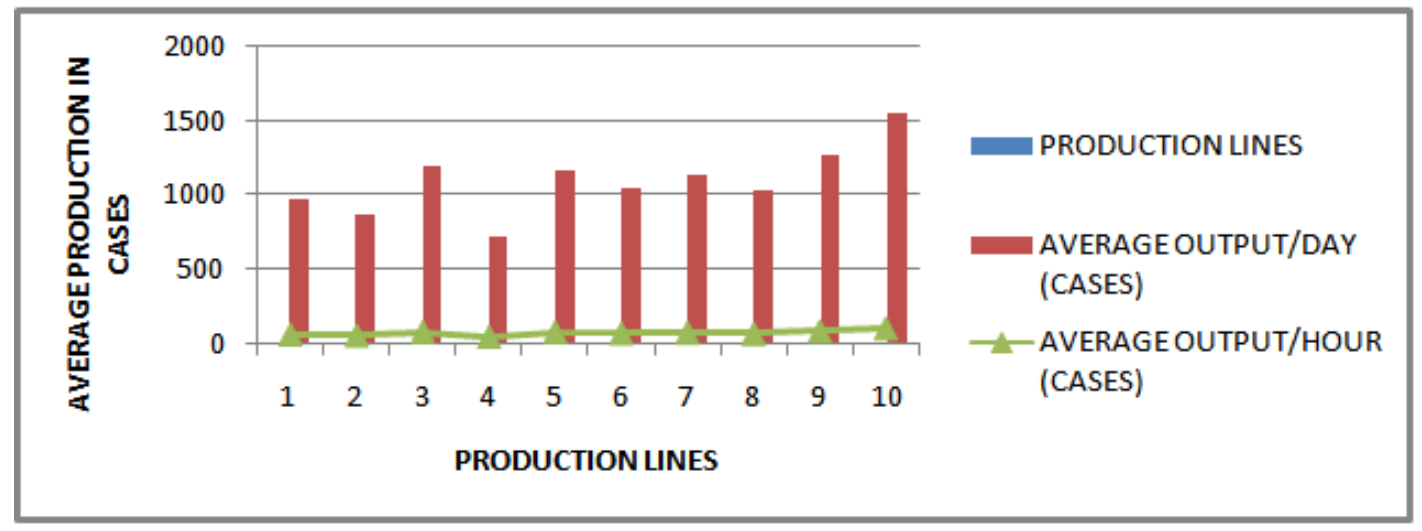

Figure 5: Average Production for the Month of October 2016

Figures 4 and 5 also present the plot of set target/output and the average production result for the line activities for 27 days for the month of October 2017. Line 10 performed better, though still below expected target. Frequent breakdown from the machines, high rate of stoppages for quality checks, and unnecessary waiting for one material or the other contributed to this general poor performance of the lines.

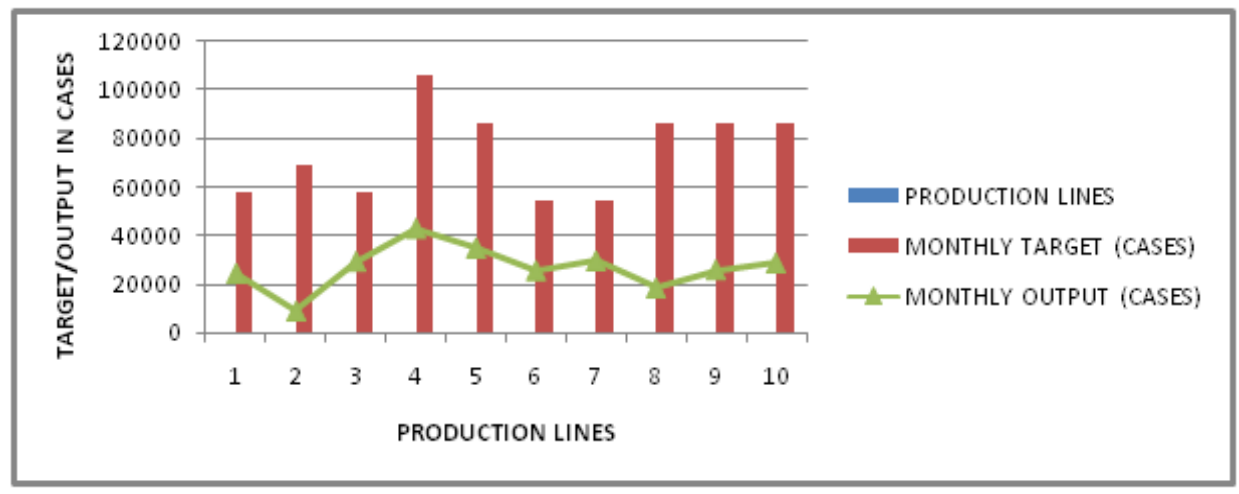

Figure 6: Expected Target/Output for Each Line for the Month of November 2016 


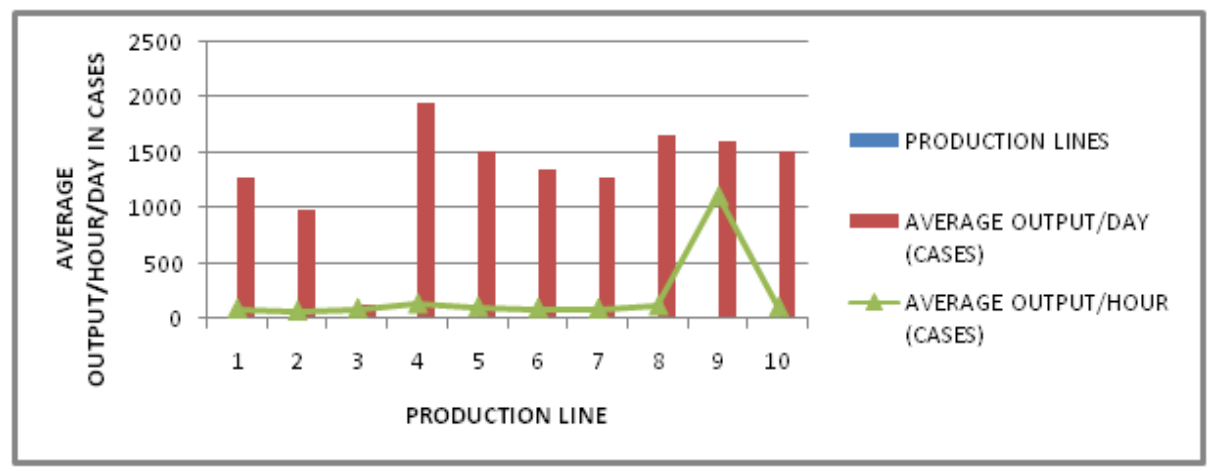

Figure 7: Average Production for November 2016

Figures 6 and 7 are the respective plots of expected target/output and the average production for the month. In this case, production was run for 25 days without break for a 2 shift of 12 hours per shift. Line 9 was found to give the highest output per hour. Though still below output but performed better than the others, because of the consistency in machine performance. Other lines could not meet up due to some inherent challenges in processes and material challenges.

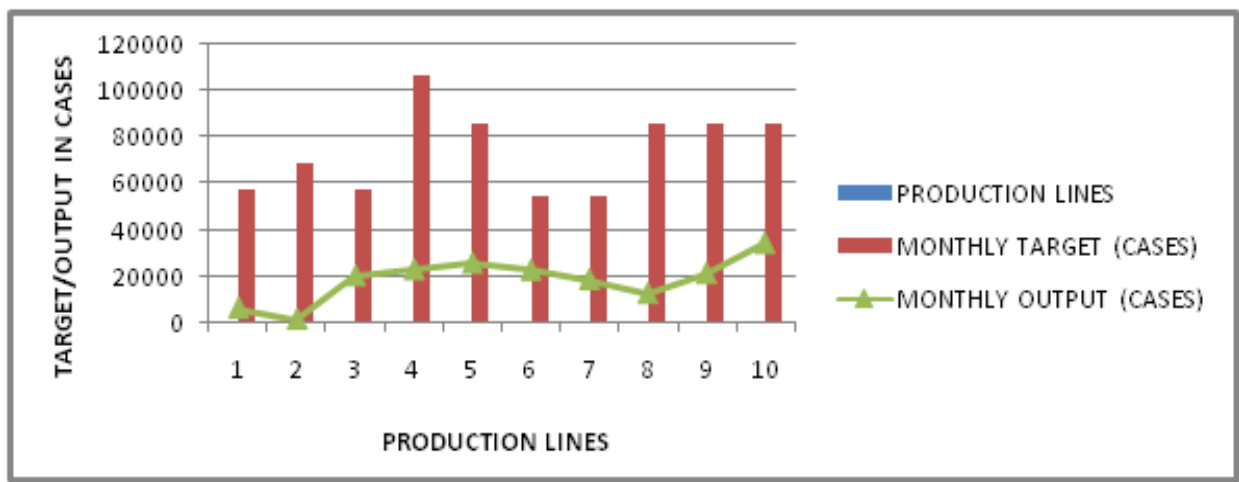

Figure 8: Expected Target/Output for Each Line for the Month of December 2016

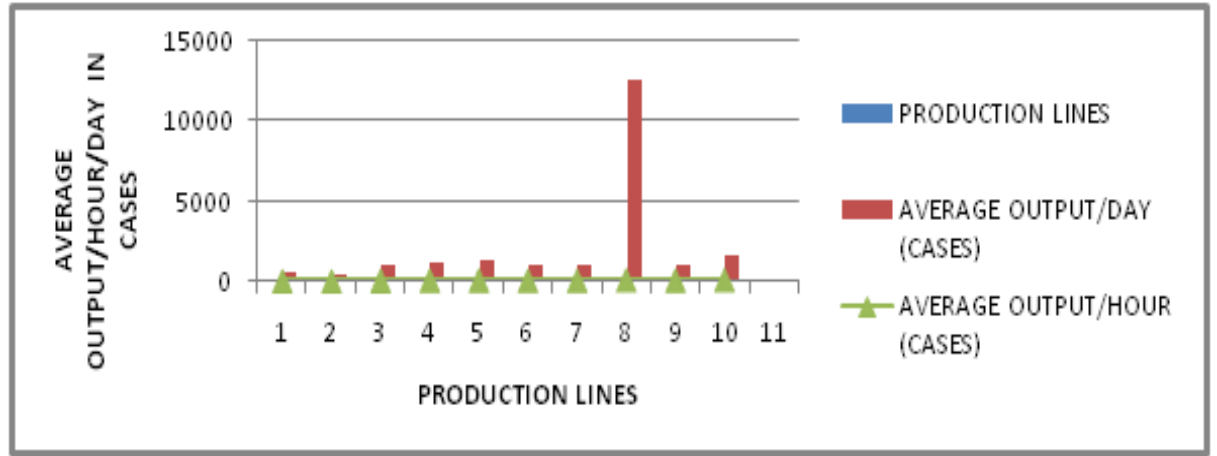

Figure 9: Average Production Result Plot for December 2016

More so, figures 8 and 9 shows the result of production activities for the month of December 2016. The production was equally run for 25 days of 2 shifts of 12 hours each. Line 8 was found to have a maximum average production output of 108.4 cases per hour. Every other line had low output. It was also discovered that machine frequent breakdown and material problem contributed immensely to the entire performance of the lines. 


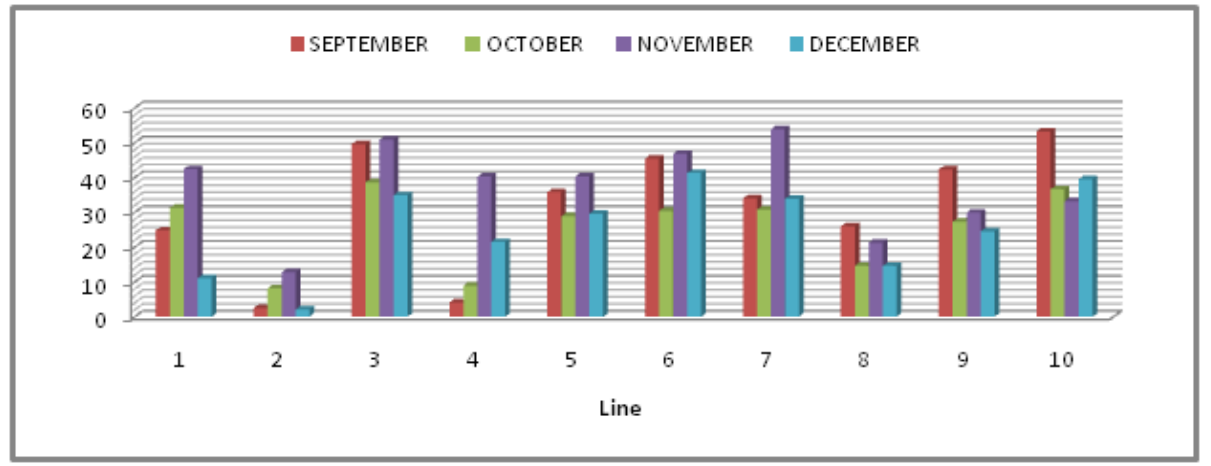

Figure 10: Comparison of their Percentage Output Delivered for Each Line and Month

Figure 10 is a plot of the percentage performances of each of the lines in each month. From the plot, it can be deduced that lines 3 and line 7 performed up to half of the set target with a values of $50 \%$ and $53.9 \%$ respectively in the month of November. The machines actually had less of stoppages but processes were interrupted for quality checks which led to time wastage. Line 10 also met $50 \%$ of the set target in the month of September. It can also be deduced from the plot that apart from the lines which met up to $50 \%$ in there set target, every other line performed below output in all the months in which study was carried out.

\section{CRITICAL OBSERVATIONS AND SUGGESTION OF POSSIBLE MEASURES}

Machine Failures: The machines have similar design and principles of operation apart from the fact that they perform different functions. When a breakage is observed on any of the machines, it is important to locate the spot where such occurrence took place, that is at the worm and guide or between the star wheel and worm because these parts have been synchronized to move with same speed. Failure to check both the inlet and outlet star wheel to ensure that they are set will always result to losses of material such as alcohol, bottle/pet, cap and labels and low output.

Therefore materials of standard specifications must be used during operation to avoid material wastages and unnecessary machine adjustment which contributed to time wastage and low output.

Work Study: The work-man behind the machine is also important as the machine. The efficiency of the machine depend on the machine and man, therefore for productivity to be increased the operators must be efficiently trained and willing to deliver. The line leaders must ensure the best way of doing the job and time taken to do it efficiently. This can be achieved by breaking down the job into its various units thereby eliminating loitering and taking proper assessment of the time taken to do the job. The organization must ensure that all workers are trained to do it the best way, this will ensure high output, reduced losses and less cost.

Work in Progress (W I P): Another major disturbance of the smooth operation is the work in progress. Supply of material is not consistent thereby leading to machine/process stoppage which contributed a great delay. This can be reduced by giving proper progress report concerning performance during the shift, quantity of materials used, quantity remaining and the losses during the shift which should be done before handing over to the next shift. From here we can invariably track losses probably reduce them.

\section{CONCLUSIONS}

Experimental analyses of the output of beverage bottling company have been carried out in ten different lines for 
four (4) different months. The output result of these lines revealed that they have been performing far below required target. The analytical result of their percentage output delivered per month also showed that only line 3 and 7 had a performance of $50 \%$ and $53.9 \%$ in the month of November. This also implies that the company has been operating below Productivity which is referred to as operating efficiency of any production firm. For the company to improve her productivity efficiently, the input resources such as men, money, materials, machinery, time, energy and space must be monitored and equally managed well.

\section{ACKNOWLEDGEMENTS}

The authors wished to acknowledge the management of Covenant University for their part sponsorship and contribution made to the success of the completion of this research paper.

\section{REFERENCES}

1. Daan van Leer "Improving performance of the Star Bottle production line" A case study at Heineken page 3-5, December 2, 2014.

2. Rui Borges Lopes, Filipa Freitas, Inês Sousa "Application of Lean Manufacturing Tools in the Food and Beverage Industries"

3. Fawaz A. Abdulmalek, Jayant Rajgopal "Analyzing the benefits of lean manufacturing and value stream mapping via simulation" A process sector case study. International Journal of Production Economics.

4. Jafri Mohd Rohani, Seyed Mojib Zahraee "Production line analysis via value stream mapping": a lean manufacturing process of color industry. 2nd International Materials, Industrial, and Manufacturing Engineering Conference, MIMEC2015, 4-6 February 2015, Bali Indonesia.

5. Onanaye, Adeniyi S., Steady State Solution of Optimal Production Control of a Dynamic n-Product Manufacturing System with Setup Cost and Time, International Journal of Mechanical and Production Engineering Research and Development (IJMPERD), Volume 3, Issue 2, April - June 2013, pp. 33-46

6. Matgorzata jasiulewicz-kaczmarek, Tomasz Bartkowiak."Improving the performance of a filling line based on simulation" Conference Paper in IOP Conference Series Materials Science and Engineering 145(4) · June 2016

7. Mike Poland "Line Performance Optimization, a problem-solving process for pharmaceutical manufacturing" CMRP, Life Cycle Engineering page 24, May 2014.

8. M. Colledani, F. Gandola, A. Matta \& T. Tolio "Performance evaluation of linear and non-linear multi-product multi-stage lines with unreliable machines and finite homogeneous buffers" ISSN: 0740-817X (Print)1545-8830 Online Journal homepage: http://www.tandfonline.com/loi/uiie20 (2008)

9. Tomasz Bartkowiak, Pawel Pawlewski "Reducing negative impact of machine failures on performance of filling and packaging production line - a simulative study" Proceedings of the 2016 Winter Simulation Conference.

10. Sibson Dalgo Edakara "A Novel Methodology to Investigate the Performance of Production Line Systems using an Integrated Product Service System Approach "Faculty of Engineering and Physical Sciences, PhD thesis report University of Manchester 2013.

11. Riddhish Thakore, Rajat Dave, Tejas Parsana, Amit Solanki "Six Sigma Implementation Practice in Manufacturing Industries" Int. Journal of Engineering Research and Applications ISSN: 2248-9622, Vol. 4, Issue 11(Version - 4), November 2014 pp.63-69 